\title{
Management of Sacral Tumors Requiring Spino-Pelvic Reconstruction with Different Histopathologic Diagnosis: Evaluation with Four Cases
}

\author{
Murat Arıkan ${ }^{1}$, Guray Togral ${ }^{1}$, Askin Esen Hasturk ${ }^{2}$, Fevzi Kekec ${ }^{1}$, Murat Parpucu ${ }^{3}$, Safak Gungor ${ }^{1}$ \\ ${ }^{1}$ Department of Orthopedics, Oncology Training and Research Hospital, Ankara, Turkey \\ ${ }^{2}$ Department of Neurosurgery, Oncology Training and Research Hospital, Ankara, Turkey \\ ${ }^{3}$ Department of Anesthesiology, Oncology Training and Research Hospital, Ankara, Turkey
}

In this retrospective study, surgical results of four patients with sacral tumors having disparate pathologic diagnoses, who were treated with partial or total sacrectomy and lumbopelvic stabilization were abstracted. Two patients were treated with partial sacral resection and two patients were treated with total sacrectomy and spinopelvic fixation. Fixation methods included spinopelvic fixation with rods and screws in two cases, reconstruction plate in one case, and fresh frozen allografts in two cases. Fibular allografts used for reconstruction accelerated bony union and enhanced the stability in two cases. Addition of polymethyl methacrylate in the cavity in the case of a giant cell tumor had a positive stabilizing effect on fixation. As a result, we can conclude that mechanical instability after sacral resection can be stabilized securely with lumbopelvic fixation and polymethyl methacrylate application or addition of fresh frozen allografts between the rods can augment the stability of the reconstruction.

Keywords: Spinopelvic reconstruction; Sacrectomy; lliac screw fixation; Fresh frozen fibular allograft

\section{Introduction}

Primary sacral tumors are rare, accounting for $7 \%$ of all spinal tumors [1]. They can arise from a neoplastic nidus in bone, cartilage, or soft tissue (neural tissue, meningeal tissue, or embryonic rests) [2]. Most sacral tumors are benign aggressive lesions such as aneurysmal bone cyst, osteoblastoma, and giant cell tumor, or low-grade malignancies such as chordoma or chondrosarcoma [3].

Sacral tumors are usually diagnosed late and often present as large, advanced neoplastic masses because of mild initial symptoms [4]. The local recurrence rate of sacral tumors is relatively high due to complex anatomy and advanced disease. Intraregional curettage, together with cryo or thermo cauterization may provide a complete cure for benign lesions. In contrast, wide resection is necessary for cure or long-term disease-free control in radio- and chemo-resistant malignancies [5].

Large sacral neoplasms require technically difficult and time-consuming operations. Fatal blood loss during the operation and wound complications make sacral tumor resection challenging [6]. Tumor resection with wide

Received Feb 4, 2015; Revised Feb 27, 2015; Accepted Feb 28, 2015

Corresponding author: Murat Arıkan

Department of Orthopedics, Oncology Training and Research Hospital,

Vatan Caddesi No.33 Demetevler, 06200 Ankara, Turkey

Tel: +90-312-336-09-09, Fax: +90-312-336-96-81, E-mail: smuratark@yahoo.com 
margins can result in lumbopelvic instability and loss of neurologic functions while performing total or subtotal sacrectomy. Partial sacrectomy operations are usually well tolerated without requiring stabilization, but in patients with extensive expansion of the tumor requiring total sacrectomy, the vertical and rotational instability necessitates stabilization between the lumbar spine and the pelvis to allow for mobilization and improved function [7]. The clinical results are affected by the extent of sacral resection and the complexity of the amputation, mechanical and neurological consequences, the oncological margins, and the possible occurrence of local and systemic complications $[8,9]$.

\section{Case Reports}

Four cases of sacral tumor underwent sacral resection and follow-up spinopelvic reconstruction procedure was performed respectively. One male patient and three female patients with a mean age of 35 years (range, 27-45 years) were included in the study. Patients had pain for a median time period of 11 months (range, 3 months-2 years). The mean follow-up time was 43.7 months (range, 19-72 months) and none of the patients died during this period or had metastases to the lungs. Pain was most frequently noted in the lower back and it commonly radiated into the thighs. Additional symptoms included bowel or bladder incontinence, muscle weakness, perineal hypoesthesia, and erectile dysfunction. Radiographs, computerized tomography (CT) scans, and magnetic resonance imaging (MRI) were performed in all patients. The histopathologic diagnosis was confirmed with CT-guided needle biopsy and it was diagnostic in only three patients, with one pa- tient (subependymoma) requiring open incisional biopsy because of inadequate specimen with CT-guided biopsy.

Total sacrectomy was performed in two patients with total removal of the tumor and surrounding soft tissue in the patients with subependymoma and malignant giant cell tumor as the lesion was recurrent and large. Partial sacrectomy with a one stage procedure via a posterior approach was preferred in the other two patients. In patients with chordoma and subependymoma, reconstruction was implemented with polyaxial screws, rods, and fresh frozen fibular allografts. No allograft-related complication, recurrence, or hardware failure was recorded during the clinical follow-up.

\section{Case 1}

A 45-year-old female patient was referred from another center where she underwent curettage and bone grafting for the diagnosis of giant cell tumor at the S1-3 level. Patient was admitted to our clinic with local recurrence that developed 6 months after the operation and additional operation was planned in our clinic. Additional CT-guided needle biopsy revealed a low-grade malignant giant cell tumor at the S1-3 level. Coronal post-contrast T2 and sagittal T2 MRI images showed heterogeneous intermediate-to-high signal densities within the lesion and low signals at the periphery, indicating recurrence of the tumor localized at the S1-3 level (Fig. 1A, B). Preoperative arterial embolization was performed in order to decrease intraoperative bleeding. Total sacrectomy was performed via the anterior-posterior combined approach along with exploration and preservation of major vessels; a temporary colostomy was also performed in order to
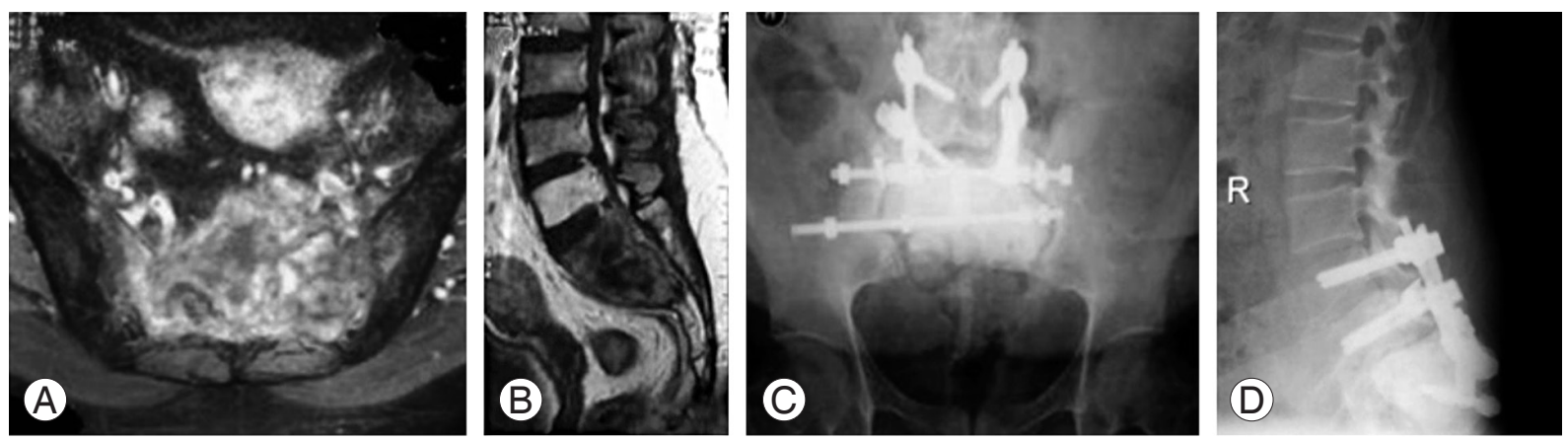

Fig. 1. (A, B) Coronal post contrast $T 2$ and sagittal T2 magnetic resonance images of malignant giant cell tumor located at the S1-3 level. (C) Anteroposterior X-ray at the 5-year follow-up. The defect is filled with polymethyl methacrylate after total sacrectomy and spinoiliac stabilization is achieved with 2 rods in order to provide stable fixation. (D) Lateral X-ray showing reconstruction and polymethyl methacrylate. 
avoid rectal injury followed by replenishment of the defect formed below the L5 level (sacral space) with polymethyl methacrylate (PMMA) as seen on an anteroposterior (AP) $\mathrm{X}$-ray (Fig. 1C, D). Total sacrectomy was preferred as the case showed malignant degeneration and extensive soft tissue involvement with a possible risk of lumbopelvic instability after resection at the S1-3 level. S1 nerve root was preserved but $\mathrm{S} 2-3$ nerve roots were sacrificed as they were within the resection field resulting in anal sphincter dysfunction after closure of the colostomy. Superficial wound infection was also observed after 1 week of operation. After serial surgical debridement and intravenous antibiotic therapy, infection was controlled. At the 5-year follow-up, the patient has no evidence of disease except for residual sphincter dysfunction.

\section{Case 2}

A 38-year-old male patient presented with pain and motor weakness in the right extremity since 8 months. The diagnosis of chordoma localized at the S2-3 level was confirmed with radiologic and histopathologic examinations. Coronal T2 and sagittal post-contrast T2 MRI showed a destructive heterogeneous high signal on T2-weighted images located at the neighboring left sacroiliac joint (Fig. 2A, B). A CT guided needle biopsy was compatible with chordoma at the S2-3 level. The patient underwent partial sacrectomy using a single stage posterior approach combined with lumbopelvic fixation which was augmented with fresh frozen fibular allografts in order to improve the stabilization with new bone formation. One lumbopelvic rod was attached with transpedicular screws. İntra operatively, S2-3 nerve roots were sacrificed as the sacral canal was infiltrated. The surgical margins were clear on histopathologic examination. An AP radiograph obtained after 6 years of follow-up after surgery revealed that there was no recurrence and the reconstruction with fibular allografts and spinoiliac stabilization with 2 rods was stable. Consolidation of the grafts was observed radiologically (Fig. 2C, D). The patient is ambulatory with permanent anal sphincter dysfunction and had no evidence of recurrent disease in the sixth year of follow-up.

\section{Case 3}

A 27-year-old female patient with a diagnosis of schwannoma localized at the S2-3 level was referred to our clinic after initial surgical resection in another hospital. She underwent marginal resection of the sacral schwannoma via a posterior approach but had recurrence after 6 months. MRI showed heterogeneous signal intensity with bony destruction on coronal post contrast $\mathrm{T} 2$ and sagittal T2 images with soft tissue extension indicating a malignant pathology (Fig. 3A, B). The CT guided needle biopsy revealed a malignant schwannoma necessitating a more radical operation. As the lesion was located below the S1 level, partial sacrectomy below the S1 level and spinoiliac stabilization with 2 rods and 2 transverse connections in order to achieve stable fixation were performed. The patient had complete relief of pain after surgery but urinary sphincter dysfunction was observed due to intraoperative injury to the S2-3 nerve root. AP and lateral radiographs obtained in the second year of followup showed stable reconstruction with 2 rods and 2 trans-
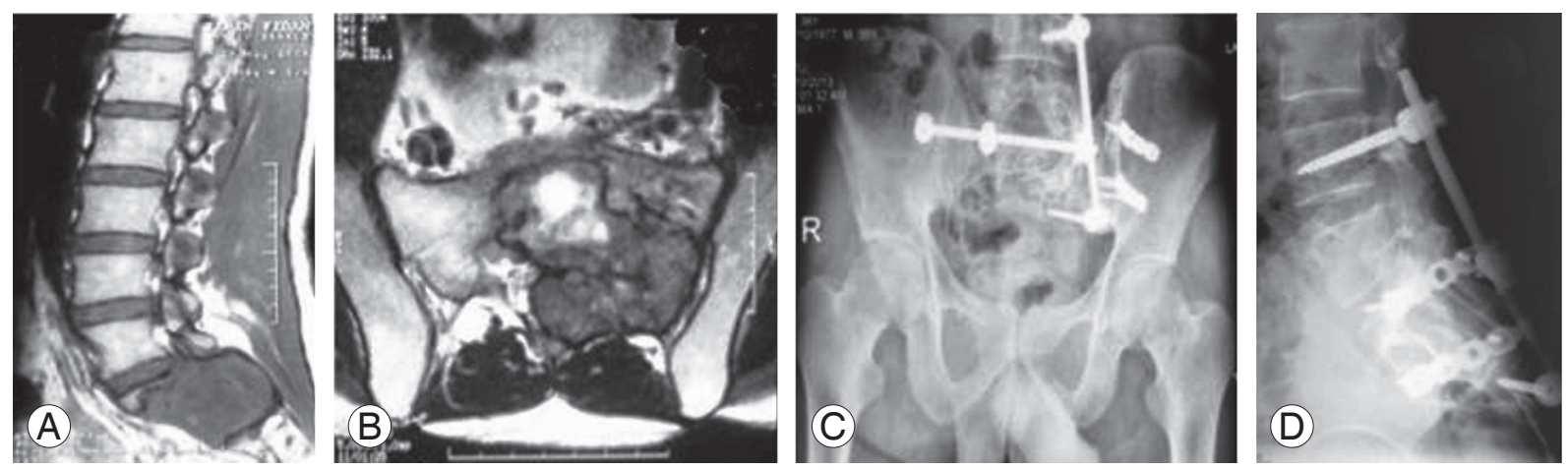

Fig. 2. (A, B) Sagittal T2 and coronal post-contrast T2 magnetic resonance images of chordoma located at the S2-3 level extending through the sacroiliac joint. (C) Anteroposterior X-ray at the 6-year follow-up. The defect is reconstructed with fibular allografts and spinoiliac stabilization after partial sacrectomy with 2 rods in order to achieve stable fixation, and consolidation of grafts is observed. (D) The lateral X-ray at the 6-year follow-up showing stable fixation and bone consolidation. 
verse connections (Fig. 3C, D). She had no recurrence after the operation and was ambulatory with no evidence of disease.

\section{Case 4}

A 30-year-old female patient was admitted to our department with lower back pain and partial motor deficit in the lower extremities. X-rays showed an irregular lytic mass at S1-2 levels extending through the fourth lumbar vertebra. Coronal post contrast T2 and sagittal T2 MRI sequences revealed a heterogeneous hyperintense lesion with increased enhancement within the central canal from S1, destroying S1-2 and extending upward within the spinal canal to the L4 vertebra (Fig. 4A, B). The diagnosis of subependymoma was confirmed with open incisional biopsy as needle biopsy was not diagnostic. The patient was treated with total sacrectomy via a combined anterior and posterior approach because of the involvement of the whole sacral bone through the sacroiliac joint and ligation of the internal iliac vessels through the anterior abdominal wall was the initial step in the surgery as the lesion was huge and destructive. The lesion extended into the

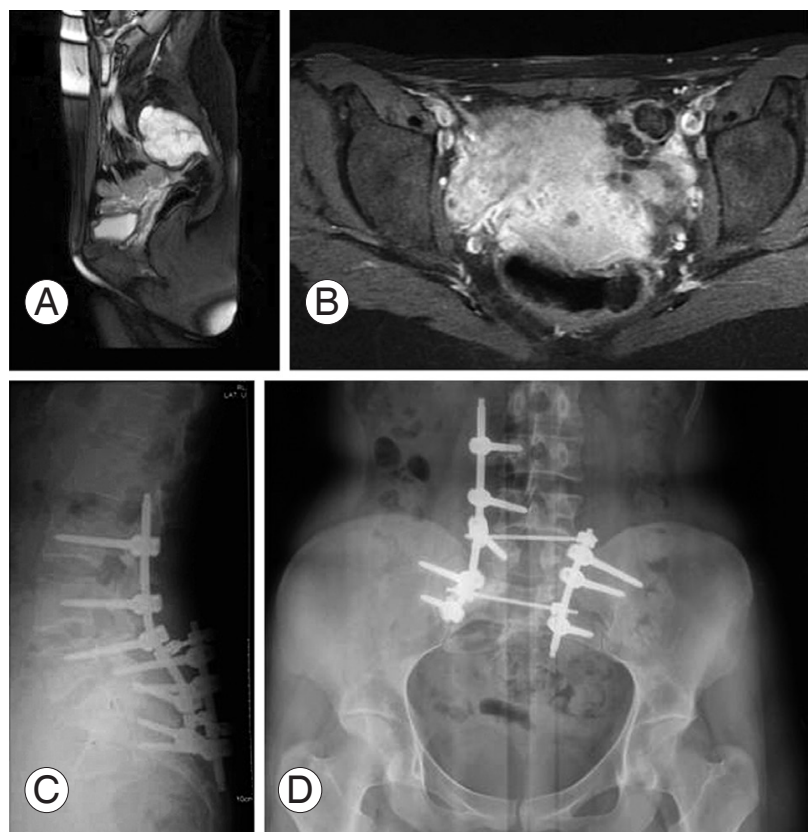

Fig. 3. (A, B) Sagittal post contrast $T 2$ and coronal T2 magnetic resonance images of schwannoma located at the $S 2-3$ level showing soft tissue extension. (C) Lateral and (D) anteroposterior X-rays at the 2-year follow-up after partial sacrectomy and spinoiliac stabilization with 2 rods and two transverse connections in order to achieve stable fixation. spinal canal up to the level of L4 vertebra, and excision of the neural tumor was extended through the L3-5 level. S2-3 nerve roots were damaged partially as they were involved by the tumor tissue resulting in anal and urinary incontinence. Also, as the S1 nerve root was invaded by the tumor tissue, nerve root dissection was performed. Spinoiliac stabilization from the L3-L5 vertebra to the pelvis was performed with 4 rods, transpedicular screws, and 1 transverse connecting rod a in order to achieve stable fixation, and the sacral defect was reconstructed with 2 fresh frozen fibular allografts as demonstrated in lateral and AP X-rays taken in the eleventh month postoperatively, showing a slight bony union (Fig. 4C, D). The transversely positioned fibular allografts secured the reconstruction mechanically (Fig. 4E).

The patient was irradiated locally in order to avoid a possible recurrence. In the postoperative period, urinary and bowel incontinence resolved partially with medical and physical treatment. Also, deep wound infection was noted at the operation site, and it was treated with serial debridement and skin reconstruction. She had no evidence of recurrent disease and is ambulatory with the help of a walker during the 19 months after the opera-
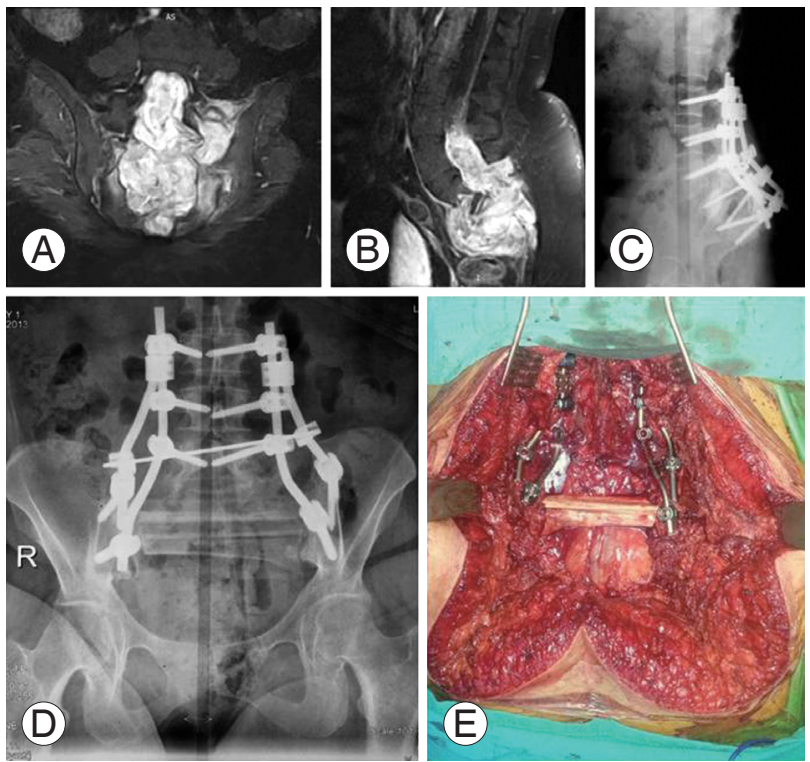

Fig. 4. (A, B) Coronal post contrast $T 2$ and sagittal T2 magnetic resonance images of sub-ependymoma located at the S1-2 level showing soft tissue extension through the L4 vertebra. (C) Lateral and (D) anteroposterior X-rays at the 19-month follow-up. Spinoiliac stabilization was achieved with 4 rods and one transverse connecting rod and the defect was reconstructed with fresh frozen fibular allografts. (E) Intraoperative image of spinopelvic reconstruction with rods, screws, and fresh frozen fibular allografts. 
tion because of residual motor deficit with slight low back pain.

\section{Discussion}

Despite the obvious advantages for disease-free survival, en bloc resection and spinopelvic reconstruction for sacral tumors pose a unique challenge for the surgeon due to the complex anatomical and biomechanical properties of the spinopelvic junction [4]. In cases two and three, asymmetrical lumbopelvic fixation was performed because of semi-destruction of the sacroiliac joint which might result in loss of strength of the construct, but clinically no mechanical deficiency due to this fixation was observed. Also, the lesions were located below the S1 level in these cases, and lumbopelvic stabilization was preferred after partial sacrectomy as lesions were extensile and showed close proximity to the S1 level in order to achieve a tumor-free wide margin. Also, the need for early ambulation and achieving a stable reconstruction necessitated this reconstruction.

Patients treated without reconstruction following a subtotal sacrectomy below the S1 level may show favorable results as major complications are infrequent following this procedure $[3,10,11]$. It is hypothesized that the muscles and scar tissue between the pelvis and spine form a biologic sling, eventually stabilizing the spine and often allowing ambulation $[12,13]$. Anyway, it is estimated that a stable reconstruction augmented either with PMMA or fibular allografts after spinopelvic fixation provided earlier ambulation and rehabilitation in the patients.

Neurologic deficits after sacral resection in cases one, two, and three were thought to be due to sacral nerve root damage during sacral resection. Case four had pre-surgical neurologic deficit in the initial referral to the hospital.

In a systematic review, Bederman et al. [4] stated that the overall instrumentation failure rate was 5 out of 31 patients (16.1\%) but, no instrument-related complications were reported in the four patients of this study. Blood loss is a significant consideration for these operations, with a mean blood loss of 9,276 mL reported in a series of 24 patients. In patients one and four, internal iliac vessels were ligated before the surgical intervention in order to decrease blood loss. With respect to this procedure, the mean blood loss in our cases was lower than expected (approximately 4,637 mL). Also, preoperative arterial embolization with gelfoam was carried out before the day of surgery in the case with giant cell tumor because of the extensile lesion and high risk for hemorrhage. In our opinion, ligation of the internal iliac vessels will be a good option for resection of aggressive benign and malignant sacral tumors with a large soft tissue component.

Surgical complications, such as superficial or deep wound infections or neurological deficits, cause significant morbidity after these complex operations. The patient with subependymoma experienced deep infection after surgery requiring additional operations for skin debridement. This condition delayed the patients' ambulation by nearly 3 months. Superficial wound infection was recorded in the case with giant cell tumor, and it was treated successfully with debridement and parenteral antibiotics.

The techniques of sacrectomy were popularized by Gunterberg et al. [14], and they suggested that resections between S-1 and S-2 caused loss of stability in approximately $50 \%$ and they also proposed that weight bearing was safe for patients after sacral resection, as long as 50\% or more of the sacroiliac joint (corresponding to at least the upper half of the S-1 segment) remained intact Cases one and four had extensive lesions beginning from the $\mathrm{S} 1$ level extending to the sacroiliac joint; therefore, sacroiliac fixation after partial sacral resection was performed in order to provide full weight bearing in the patients during the early postoperative period. As the conventional S2-3 resection does not disrupt the sacroiliac articulations and lumbopelvic stability is preserved, lumbopelvic fixation may not be required but lesions at the S1 level invading the sacroiliac joints constitute an absolute indication for total sacral resection followed by lumbopelvic stabilization in order to prevent pelvic rotation during weight bearing as in the cases with giant cell tumor and subependymoma. Lumbopelvic stabilization was preferred in cases two and three after partial sacrectomy because of the close proximity of the huge lesions to the $\mathrm{S} 1$ vertebra and provided painless and early weight bearing in the patients.

Total sacrectomy is a complex surgical procedure that results in complete dissociation of the spine and pelvis and requires specialized surgical stabilization techniques to preserve mechanical support and allow satisfactory walking ability [15]. The postoperative local recurrence rate after sacral tumor resection is directly related to the scope and degree of the resection. Factors such as age and sex had no statistically significant impact on the recurrence [16]. In a retrospective analysis of 93 cases, Zhang et 
al. [17] showed that 11 of the 37 patients had a wide margin, 11 of whom experienced a recurrence $(29.7 \%)$ and they did not report a lower recurrence rate after radical resection. No local recurrence has yet been noted in our series during a mean follow-up period of 43.7 months.

The risk of infection increases substantially in patients who have previously undergone lumbosacral surgery and in procedures with a long operation time. We reported deep and superficial wound infections sequentially in cases one and four which showed long operation times and correspondingly more bleeding. In a series in which $94 \%$ of the patients were operated on via a posterior approach with no reoperations, the infection rate was 39\% [18]. Kaiser et al. [19] reported that local recurrence rates correlated significantly with preservation of the tumor margins at the initial surgery and local recurrence rate for patients who had undergone en bloc sacral resection of chordomas without contamination of the surgical wound was as high as $28 \%$. No local recurrence was recorded among these four different primary sacral tumors as we think that surgical margins were clearly preserved after sacral resection.

In conclusions, sacral tumors treated surgically with total or partial sacrectomy and lumbopelvic stabilization show good functional outcomes and low complication rates. Sacral nerve preservation during sacrectomy, if possible, is an important point for maintaining the neurologic outcome in the patients. Preoperative embolization or intraoperative ligation of internal iliac vessels significantly decreased the blood loss compared with values reported in the literature. Fresh frozen fibular allografts and PMMA can be good alternatives for augmenting the strength of the lumbopelvic reconstruction.

\section{Conflict of Interest}

No potential conflict of interest relevant to this article was reported.

\section{References}

1. Feldenzer JA, McGauley JL, McGillicuddy JE. Sacral and presacral tumors: problems in diagnosis and management. Neurosurgery 1989;25:884-91.

2. Syed R, Bishop JA, Ali SZ. Sacral and presacral lesions: cytopathologic analysis and clinical correlates. Diagn Cytopathol 2012;40:7-13.
3. Capanna R, Briccoli A, Campanacci L. Benign and malignant tumors of the sacrum. In: Frymoyer JW, Ducker TB, editors. The adult spine: principles and practice. 2nd ed. Philadelphia: Lippincott-Raven; 1997. p.2367-405.

4. Bederman SS, Shah KN, Hassan JM, Hoang BH, Kiester PD, Bhatia NN. Surgical techniques for spinopelvic reconstruction following total sacrectomy: a systematic review. Eur Spine J 2014;23:305-19.

5. Atalar H, Selek H, Yildiz Y, Saglik Y. Management of sacrococcygeal chordomas. Int Orthop 2006;30:5148.

6. Li D, Guo W, Qu H, et al. Experience with wound complications after surgery for sacral tumors. Eur Spine J 2013;22:2069-76.

7. Hugate RR Jr, Dickey ID, Phimolsarnti R, Yaszemski MJ, Sim FH. Mechanical effects of partial sacrectomy: when is reconstruction necessary? Clin Orthop Relat Res 2006;450:82-8.

8. Sar C, Eralp L. Surgical treatment of primary tumors of the sacrum. Arch Orthop Trauma Surg 2002;122: 148-55.

9. Zileli M, Hoscoskun C, Brastianos P, Sabah D. Surgical treatment of primary sacral tumors: complications associated with sacrectomy. Neurosurg Focus 2003;15:E9.

10. Randall RL, Bruckner J, Lloyd C, Pohlman TH, Conrad EU 3rd. Sacral resection and reconstruction for tumors and tumor-like conditions. Orthopedics 2005;28:307-13.

11. Michel A. Total sacrectomy and lower spine resection for giant cell tumor: one case report. Chir Organi Mov 1990;75:117-8.

12. Ruggieri P, Angelini A, Ussia G, Montalti M, Mercuri M. Surgical margins and local control in resection of sacral chordomas. Clin Orthop Relat Res 2010;468: 2939-47.

13. Guo Y, Yadav R. Improving function after total sacrectomy by using a lumbar-sacral corset. Am J Phys Med Rehabil 2002;81:72-6.

14. Gunterberg B, Romanus B, Stener B. Pelvic strength after major amputation of the sacrum: an exerimental study. Acta Orthop Scand 1976;47:635-42.

15. Fourney DR, Gokaslan ZL. Current management of sacral chordoma. Neurosurg Focus 2003;15:E9.

16. Fourney DR, Rhines LD, Hentschel SJ, et al. En bloc resection of primary sacral tumors: classification of 
surgical approaches and outcome. J Neurosurg Spine 2005;3:111-22.

17. Zhang ZY, Fu CF, Yang YX, Wang LQ, Cui Y, Liu Y. Long-term outcomes following en bloc resection for sacral tumor: a retrospective analysis of 93 cases. Orthopedics 2011;34:e403-7.

18. Sciubba DM, Nelson C, Gok B, et al. Evaluation of factors associated with postoperative infection following sacral tumor resection. J Neurosurg Spine 2008;9:593-9.

19. Kaiser TE, Pritchard DJ, Unni KK. Clinicopathologic study of sacrococcygeal chordoma. Cancer 1984;53: 2574-8. 